\title{
TORSION IN TENSOR POWERS OF MODULES
}

\author{
OLGUR CELIKBAS, SRIKANTH B. IYENGAR, \\ GREG PIEPMEYER, AND ROGER WIEGAND
}

\begin{abstract}
Tensor products usually have nonzero torsion. This is a central theme of Auslander's 1961 paper; the theme continues in the work of Huneke and Wiegand in the 1990s. The main focus in this article is on tensor powers of a finitely generated module over a local ring. Also, we study torsion-free modules $N$ with the property that $M \otimes_{R} N$ has nonzero torsion unless $M$ is very special. An important example of such a module $N$ is the Frobenius power $p^{e} R$ over a complete intersection domain $R$ of characteristic $p>0$.
\end{abstract}

\section{$\S 1$. Introduction}

In a 1961 paper, Auslander [1] studied torsion in tensor products of nonzero finitely generated modules $M$ and $N$ over unramified regular local rings $R$. Under the assumption that $M \otimes_{R} N$ is torsion-free, he proved that

(1) $M$ and $N$ must be torsion-free, and

(2) $M$ and $N$ are Tor independent; that is, $\operatorname{Tor}_{i}^{R}(M, N)=0$ for all $i \geq 1$.

The two conclusions are cleverly intertwined in his proof, which we revisit in Section 3 of the present paper. We show, over a reduced complete intersection ring $R$ of positive characteristic $p$, that $M \otimes_{R} \varphi^{e} R$ is torsion-free if and only if $M$ is torsion-free and of finite projective dimension, in which case $\operatorname{Tor}_{i}^{R}\left(M, \varphi^{e} R\right)=0$ for all $i \geq 1$. (Here $\varphi: R \rightarrow R$ is the Frobenius endomorphism and $\varphi^{e} R$ is the module obtained from $R$ by restriction of scalars along $\varphi^{e}$.) When $R$ is $\mathrm{F}$-finite, we obtain a criterion for regularity: $R$ is regular if and only if $\left(\varphi^{e} M\right) \otimes_{R} \varphi^{e} R$ is torsion-free for some (equivalently, every) nonzero finitely generated $R$-module $M$.

Our main results are in Section 2, where we study torsion in tensor powers. We obtain detailed information on annihilators of elements in $\otimes_{R}^{n} M$ and draw several conclusions. Suppose, for example, that $\underline{r}=r_{1}, \ldots, r_{d}$ is a

Received January 27, 2014. Revised July 14, 2014. Accepted August 14, 2014.

2010 Mathematics Subject Classification. Primary 13C12; Secondary 13D07. 
regular sequence in $R$ and that $M$ is the cokernel of the $d \times 1$ matrix $[\underline{r}]^{\mathrm{t}}$. We show in Theorem 2.5 that $\otimes_{R}^{t} M$ is torsion-free if and only $t \leq d$. This result should be compared with Auslander's observation in [1, p. 638] that the same holds when $M$ is the $(d-1)$ st syzygy of a module of projective dimension $d$ over a $d$-dimensional regular local ring (see also [7, Proposition 3.1]). If $R$ is local, the "only if" direction holds much more generally: we show in Theorem 2.7 that, if we write $M$ as the cokernel of an $m \times n$ matrix $\theta$ with entries in the maximal ideal of $R$, and if some entry of $\theta$ is a nonzerodivisor, then $\otimes_{R}^{t} M$ has nonzero torsion for every $t \geq m$.

Throughout this article, $R$ is a commutative, Noetherian ring.

\section{$\S 2$. Torsion in tensor powers}

In this section we establish results on annihilators of elements in tensor powers of modules.

Notation 2.1. Given elements $\underline{m}:=m_{1}, \ldots, m_{d}$ in an $R$-module $M$, we consider the element in $\otimes_{R}^{d} M$ defined by

$$
\tau(\underline{m}):=\sum_{\sigma \in S_{d}} \operatorname{sign}(\sigma) m_{\sigma(1)} \otimes \cdots \otimes m_{\sigma(d)} .
$$

Proposition 2.2. Let $M$ be an $R$-module. If elements $m_{1}, \ldots, m_{d}$ in $M$ and $r_{1}, \ldots, r_{d}$ in $R$ satisfy

$$
r_{1} m_{1}+\cdots+r_{d} m_{d}=0
$$

then $\left(r_{1}, \ldots, r_{d}\right) \cdot \tau(\underline{m})=0$ in $\otimes_{R}^{d} M$.

Proof. The twisted shuffle product gives the graded $R$-algebra $\bigoplus_{n \geq 0} \otimes_{R}^{n} M$ a strictly skew-commutative structure (see [11, Chapter $\mathrm{X}$, (12.4)]. Strictly skew-commutative means that for any $a \in \otimes_{R}^{i} M$ and $b \in$ $\otimes_{R}^{j} M$ there are equalities

$$
a \star b=(-1)^{i j} b \star a, \quad \text { and } \quad a \star a=0 \quad \text { when } i \text { is odd. }
$$

By definition of the shuffle product, $\tau(\underline{m})=m_{1} \star \cdots \star m_{d}$. Thus, for each $j$ we have

$$
\begin{aligned}
r_{j} \cdot \tau(\underline{m}) & =m_{1} \star \cdots \star m_{j-1} \star r_{j} m_{j} \star m_{j+1} \star \cdots \star m_{n} \\
& =-\sum_{i \neq j} r_{i}\left(m_{1} \star \cdots \star m_{j-1} \star m_{i} \star m_{j+1} \star \cdots \star m_{n}\right)=-\sum_{i \neq j} r_{i} 0=0
\end{aligned}
$$


There is a "universal" source for the element $\tau(\underline{m})$ in the following sense.

REMARK 2.3. Consider the polynomial ring $\mathbb{Z}[\underline{x}]$ on indeterminates $\underline{x}:=$ $x_{1}, \ldots, x_{d}$, and let $U$ be the $\mathbb{Z}[\underline{x}]$-module with presentation

$$
0 \rightarrow \mathbb{Z}[\underline{x}] \stackrel{\left[x_{1}, \ldots, x_{d}\right]^{\mathrm{t}}}{\longrightarrow} \mathbb{Z}[\underline{x}]^{d} \rightarrow U \rightarrow 0 .
$$

Let $u_{1}, \ldots, u_{d}$ be the generators of $U$ corresponding to the standard basis for $\mathbb{Z}[\underline{x}]^{d}$, so that $x_{1} u_{1}+\cdots+x_{d} u_{d}=0$; that is, $\underline{x}$ and $\underline{u}$ satisfy (2.2.1). Then $\operatorname{ann}_{\mathbb{Z}[\underline{x}]} \tau(\underline{u}) \supseteq(\underline{x})$ by Proposition 2.2; we will see, in Theorem 2.5 below, that in fact $\operatorname{ann}_{\mathbb{Z}[\underline{x}]} \tau(\underline{u})=(\underline{x})$.

Given any $R$-module $M$ with a syzygy relation (2.2.1), consider the ring homomorphism $\mathbb{Z}[\underline{x}] \rightarrow R$ taking $x_{i}$ to $r_{i}$, for each $i$, and extending the structure homomorphism $\mathbb{Z} \rightarrow R$. The hypothesis on $M$ implies that there is a homomorphism of $\mathbb{Z}[\underline{x}]$-modules

$$
f: U \rightarrow M \text { with } f\left(u_{i}\right)=m_{i} \text { for } i=1, \ldots, d .
$$

Under the induced map $\otimes^{d} f: \otimes_{\mathbb{Z}[\underline{x}]}^{d} U \rightarrow \otimes_{R}^{d} M$, the element $\tau(\underline{u})$ maps to $\tau(\underline{m})$.

This remark prompts the discussion below, culminating in Theorem 2.5. First we review some notions regarding depth. For details, see [4, Chapter 1].

\subsection{Depth}

Let $M$ be a finitely generated $R$-module, and let $I$ be an ideal of $R$ satisfying $I M \neq M$. The $I$-depth of $M$ is the number

$$
\operatorname{depth}_{R}(I, M)=\inf \left\{n \geq 0 \mid \operatorname{Ext}_{R}^{n}(R / I, M) \neq 0\right\} .
$$

The $I$-depth of $M$ is always finite and is equal to the length of every maximal $M$-regular sequence in $I$.

If $\underline{x}:=x_{1}, \ldots, x_{d}$ is a sequence of elements in $R$, and if $K$ is the Koszul complex on $\underline{x}$, then the $(\underline{x})$-depth of $M$ may be computed from its Koszul homology:

$$
\operatorname{depth}_{R}((\underline{x}), M)=d-\sup \left\{i \geq 0 \mid \mathrm{H}_{i}\left(K \otimes_{R} M\right) \neq 0\right\} .
$$

This is the depth sensitivity of the Koszul complex.

Suppose now that $\underline{x}$ is $R$-regular. Then $K$ is a free resolution of $R /(\underline{x})$, and hence $\mathrm{H}_{*}\left(K \otimes_{R} M\right) \cong \operatorname{Tor}_{*}^{R}(R /(\underline{x}), M)$. In this case, we have

$$
\operatorname{depth}_{R}((\underline{x}), M)=d-\sup \left\{i \geq 0 \mid \operatorname{Tor}_{i}^{R}(R /(\underline{x}), M) \neq 0\right\} .
$$

If $R$ is local with maximal ideal $\mathfrak{m}$, we write $\operatorname{depth}_{R} M$ for the $\mathfrak{m}$-depth of $M$ and call it the depth of $M$. 


\subsection{A Koszul syzygy module}

Let $R$ be a Noetherian ring, and let $\underline{r}:=r_{1}, \ldots, r_{d}$ be a regular sequence in $R$ with $(\underline{r}) \neq R$. Consider the complex

$$
F:=0 \rightarrow R \stackrel{\left[r_{1}, \ldots, r_{d}\right]^{\mathrm{t}}}{\longrightarrow} R^{d} \rightarrow 0
$$

concentrated in degrees 0 and 1 . Set $M=\mathrm{H}_{0}(F)$; as $r_{1}$ is a nonzerodivisor, $F$ is a free resolution of $M$.

Lemma 2.4. Let $M, d$, and $F$ be as in Section 2.2. For each $n=1, \ldots, d$, the following statements hold:

(1) $M$ and $\otimes_{R}^{n-1} M$ are Tor independent; and

(2) $\otimes_{R}^{n} F$ is a free resolution of $\otimes_{R}^{n} M$, and $\operatorname{pd}_{R}\left(\otimes_{R}^{n} M\right)=n$.

Proof. The base case is $n=1$, and then (1) and (2) are clear. Fix an integer $n$ with $2 \leq n \leq d$, and assume that these statements hold for all integers $\leq n-1$. Set $I=(\underline{r})$. Since $\otimes_{R}^{n-1} F$ is a free resolution of $\otimes_{R}^{n-1} M$, we have

$$
\operatorname{Tor}_{*}^{R}\left(R / I, \otimes_{R}^{n-1} M\right)=\mathrm{H}_{*}\left((R / I) \otimes_{R}\left(\otimes_{R}^{n-1} F\right)\right) \cong\left(\otimes_{R}^{n-1}\left((R / I) \otimes_{R} F\right)\right)_{*},
$$

where the last isomorphism holds because the complex in question has zero differential. In particular, $\operatorname{Tor}_{n-1}^{R}\left(R / I, \otimes_{R}^{n-1} M\right) \cong R / I \neq 0$, so that

$$
\sup \left\{i \geq 0 \mid \operatorname{Tor}_{i}^{R}\left(R /(\underline{r}), \otimes_{R}^{n-1} M\right) \neq 0\right\}=n-1 .
$$

We can now complete the induction step.

(1) The induction hypothesis implies that $\otimes_{R}^{n-1} F$ is a free resolution of $\otimes_{R}^{n-1} M$, so (2.4.1) and (2.3.1) show that

$$
\operatorname{depth}_{R}\left(I, \otimes_{R}^{n-1} M\right)=d-(n-1) \geq 1 .
$$

Moreover, $\operatorname{Tor}_{*}^{R}\left(M, \otimes_{R}^{n-1} M\right)$ is the homology of the complex

$$
F \otimes_{R}\left(\otimes_{R}^{n-1} M\right): 0 \rightarrow \otimes_{R}^{n-1} M \stackrel{[\underline{[r}]^{t}}{\longrightarrow}\left(\otimes_{R}^{n-1} M\right)^{d} \rightarrow 0
$$

(concentrated in degrees 0 and 1). By (2.4.2), some $r_{i}$ is a nonzerodivisor on $\otimes_{R}^{n-1} M$, and it follows that $M$ and $\otimes_{R}^{n-1} M$ are Tor independent.

(2) By hypothesis, $F$ and $\otimes_{R}^{n-1} F$ are free resolutions of $M$ and $\otimes_{R}^{n-1} M$, respectively. We have already proved, in (1), that these modules are Tor independent, so the complex $F \otimes_{R}\left(\otimes_{R}^{n-1} F\right)$, that is, $\otimes_{R}^{n} F$, is a free resolution of $\otimes_{R}^{n} M$. In particular, $\operatorname{pd}_{R}\left(\otimes_{R}^{n} M\right) \leq n$; that the equality holds follows from (2.4.1). 


\subsection{Torsion submodule}

Let $\mathrm{Q}(R)$ be the total quotient ring of $R$. The torsion submodule $\top_{R} M$ of $M$ is the kernel of the natural homomorphism $M \rightarrow \mathrm{Q}(R) \otimes_{R} M$. The inclusion $\top_{R} M \subseteq M$ gives rise to an exact sequence

$$
0 \rightarrow \top_{R} M \rightarrow M \rightarrow \perp_{R} M \rightarrow 0 .
$$

The module $M$ is torsion if $\top_{R} M=M$ (i.e., $M_{\mathfrak{p}}=0$ for each $\mathfrak{p} \in \operatorname{Ass}(R)$ ), and $M$ is torsion-free if $\top_{R} M=0$. Thus, $M$ is torsion-free if and only if $\bigcup \operatorname{Ass} M \subseteq \bigcup \operatorname{Ass} R$. The stronger condition, that Ass $M \subseteq$ Ass $R$, is therefore a sufficient condition for $M$ to be torsion-free. We will invoke this criterion twice in the proof of the next theorem.

Part (1) of the next result is reminiscent of Auslander's discussion in [1, p. 638] (see also [7, Proposition 3.1]).

THEOREM 2.5. Let $M$ and $\underline{r}$ be as in Section 2.2. The following statements hold:

(1) $\otimes_{R}^{n} M$ is torsion-free if and only if $n \leq d-1$;

(2) the element $\tau(\underline{m})$ in $\otimes_{R}^{d} M$ satisfies $\operatorname{ann}_{R} \tau(\underline{m})=(\underline{r})$; and

(3) the map $R /(\underline{r}) \rightarrow \otimes_{R}^{d} M$ of $R$-modules with $1 \mapsto \tau(\underline{m})$ induces a splitting

$$
\otimes_{R}^{d} M \cong(R /(\underline{r})) \oplus W,
$$

where $W$ is torsion-free; in particular, we have

$$
\operatorname{Hom}_{R}\left(R /(\underline{r}), \otimes_{R}^{d} M\right)=R \tau(\underline{m}) \neq 0 .
$$

Proof. Set $I=(\underline{r})$, let $n \leq d-1$, and fix a prime $\mathfrak{p} \in \operatorname{Ass}\left(\otimes_{R}^{n} M\right)$. If $I \subseteq \mathfrak{p}$, it follows from Lemma 2.4 that $\left(\otimes_{R}^{n} F\right)_{\mathfrak{p}}$ is a minimal free resolution of $\left(\otimes_{R}^{n} M\right)_{\mathfrak{p}} ;$ therefore,

$$
\operatorname{depth}_{R_{\mathfrak{p}}}\left(\otimes_{R}^{n} M\right)_{\mathfrak{p}}=\operatorname{depth} R_{\mathfrak{p}}-n \geq d-n \geq 1
$$

which is a contradiction. Thus, $I \nsubseteq \mathfrak{p}$, and then the $R_{\mathfrak{p}}$-module $M_{\mathfrak{p}}$ is a nonzero free module; hence, so is $\left(\otimes_{R}^{n} M\right)_{\mathfrak{p}}$. Therefore, $\operatorname{depth} R_{\mathfrak{p}}=$ $\operatorname{depth}_{R_{\mathfrak{p}}}\left(\otimes_{R}^{n} M\right)_{\mathfrak{p}}$. We have shown that $\operatorname{Ass}\left(\otimes_{R}^{n} M\right) \subseteq \operatorname{Ass} R$, and hence that $\otimes_{R}^{n} M$ is torsion-free. The "only if" direction of (1) will follow from (3).

For (2) and (3), by construction $r_{1} m_{1}+\cdots+r_{d} m_{d}=0$, so Proposition 2.2 gives an inclusion $I \subseteq \operatorname{ann}_{R} \tau(\underline{m})$. The reverse inclusion will follow, once we ascertain that the map in (3) splits. Consider the homomorphisms of $R$ modules 


$$
\begin{aligned}
\otimes_{R}^{d}\left(F_{0}\right) & \rightarrow \otimes_{R}^{d} M \rightarrow\left(\otimes_{R}^{d} M\right) \otimes_{R} R / I \cong \mathrm{H}_{0}\left(\left(\otimes_{R}^{d} F\right) \otimes_{R} R / I\right) \\
& =\otimes_{R}^{d}\left(F_{0} \otimes_{R} R / I\right),
\end{aligned}
$$

where the surjections are the natural ones; the isomorphism holds because $\otimes_{R}^{d} F$ is a free resolution of $\otimes_{R}^{d} M$, and the equality holds because the differential on $F$ has its image in $I F$. Let $\underline{e}=e_{1}, \ldots, e_{d}$ be the standard basis for $F_{0}=R^{d}$, in Section 2.2, and let $\underline{e}^{\prime}$ be the induced basis of the free $R / I$ module $F_{0} \otimes_{R} R / I$. Under the composite map, the element $\tau(\underline{e})$ maps to $\tau\left(\underline{e}^{\prime}\right)$, and $\left\{\tau\left(\underline{e}^{\prime}\right)\right\}$ extends to a basis of the $R / I$-module $\otimes_{R}^{d}\left(F_{0} \otimes_{R}(R / I)\right)$. Since $\tau(\underline{e})$ maps to $\tau(\underline{m})$ in $\otimes_{R}^{d} M$, the map in (2) splits and gives a decomposition

$$
\otimes_{R}^{d} M \cong(R / I) \oplus W
$$

It remains to verify that $W$ is torsion-free; given the decomposition above, the other parts of (3) are a consequence of this fact.

For $\mathfrak{p} \in \operatorname{Spec} R$ with $I \nsubseteq \mathfrak{p}$, the $R_{\mathfrak{p}}$-module is $M_{\mathfrak{p}}$ free, and hence so is $W_{\mathfrak{p}}$. Assume now that $I \subseteq \mathfrak{p}$. The Koszul complex on $\underline{r}$, viewed as elements in $R_{\mathfrak{p}}$, is a minimal resolution of $(R / I)_{\mathfrak{p}}$, and so it is a direct summand of $\left(\otimes_{R}^{n} F\right)_{\mathfrak{p}}$, the minimal free resolution of $\left(\otimes_{R}^{n} M\right)_{\mathfrak{p}}$. The ranks of the free modules in the top degree, $d$, of these complexes coincide (and equal 1), whence $\operatorname{pd}_{R_{\mathfrak{p}}} W_{\mathfrak{p}} \leq d-1$ and

$$
\operatorname{depth}_{R_{\mathfrak{p}}} W_{\mathfrak{p}}=\operatorname{depth} R_{\mathfrak{p}}-\operatorname{pd}_{R_{\mathfrak{p}}} W_{\mathfrak{p}} \geq 1
$$

These observations show that Ass $W \subseteq \operatorname{Ass} R$, so $W$ is torsion-free as claimed.

\subsection{Local rings}

Next we focus on local rings, where the preceding results can be strengthened to some extent.

LEMMA 2.6. Let $M$ be a finitely generated module over a local ring $(R, \mathfrak{m})$, and let $m_{1}, \ldots, m_{d} \in M$. If the images of $\left\{m_{1}, \ldots, m_{d}\right\}$ in $M / \mathfrak{m} M$ are linearly independent, then $\tau(\underline{m})$ is not in $\mathfrak{m}\left(\otimes_{R}^{d} M\right)$.

Proof. Let $m_{i}^{\prime}$ be the image of $m_{i}$ in the $k$-vector space $M / \mathfrak{m} M$. Since $\left\{m_{1}^{\prime}, \ldots, m_{d}^{\prime}\right\}$ is linearly independent, $\tau\left(\underline{m}^{\prime}\right) \neq 0$. Hence, $\tau(\underline{m}) \notin \mathfrak{m}\left(\otimes_{R}^{d} M\right)$. 
Given an $R$-module $M$, we write $I(M)$ for the ideal $\left(r_{i j}\right)$ defined by the entries in a matrix in some minimal presentation

$$
R^{\mu} \stackrel{\left[r_{i j}\right]}{\longrightarrow} R^{\nu} \rightarrow M \rightarrow 0 \quad \text { where } \nu=\nu_{R}(M) .
$$

This ideal is independent of the presentation. Moreover, $I(M)$ contains a nonzerodivisor if and only if, over $\mathrm{Q}(R)$, the total quotient ring of $R$, the module $\mathrm{Q}(R) \otimes_{R} M$ can be generated by fewer than $\nu$ elements. To see this we note that, since $\mathrm{Q}(R)$ is semilocal, the module $\mathrm{Q}(R) \otimes_{R} M$ needs $\nu$ generators if and only if $\nu_{R_{\mathfrak{p}}} M_{\mathfrak{p}}=\nu$ for some $\mathfrak{p} \in$ Ass $R$; moreover, $\nu_{R_{\mathfrak{p}}} M_{\mathfrak{p}}=\nu$ if and only if the presentation remains minimal when localized at $\mathfrak{p}$, that is, if and only if $I(M) \subseteq \mathfrak{p}$. Thus, $\mathrm{Q}(R) \otimes_{R} M$ needs $\nu$ generators if and only if $I(M) \subseteq \mathfrak{p}$ for some $\mathfrak{p} \in$ Ass $R$, that is to say, if and only if $I(M)$ consists of zerodivisors.

Recall that $M$ is said to have rank $r$ if $\mathrm{Q}(R) \otimes_{R} M$ is free over $\mathrm{Q}(R)$ of rank $r$ (see [4, Proposition 1.4.3] for different characterizations of this property).

THEOREM 2.7. Let $R$ be a local ring, and let $M$ be a nonzero finitely generated $R$-module satisfying one of the following conditions:

(1) $I(M)$ contains a nonzerodivisor; in this case, set $b=\nu_{R}(M)$; or

(2) $M$ has rank; in this case, set $b=\operatorname{rank}_{R}(M)+1$.

If $M$ is not free, then for each nonzero finitely generated $R$-module $N$ one has

$$
\top_{R}\left(\left(\otimes_{R}^{n} M\right) \otimes_{R} N\right) \neq 0 \quad \text { for each } n \geq b .
$$

Proof. It suffices to prove the statement for $n=b$, since

$$
\left(\otimes_{R}^{n} M\right) \otimes_{R} N \cong\left(\otimes_{R}^{b} M\right) \otimes_{R}\left(\left(\otimes_{R}^{n-b} M\right) \otimes_{R} N\right)
$$

and $N \neq 0$ implies that $\left(\otimes_{R}^{i} M\right) \otimes_{R} N \neq 0$ for each $i \geq 0$, by Nakayama's lemma.

(1) Let $m_{1}, \ldots, m_{b}$ be a minimal generating set for the $R$-module $M$. The element $\tau(\underline{m})$ in $\otimes_{R}^{b} M$ is annihilated by $I(M)$, by Proposition 2.2, and is not in $\mathfrak{m}\left(\otimes_{R}^{b} M\right)$, by Lemma 2.6. It follows that, for each $x$ in $N \backslash \mathfrak{m} N$, the element $\tau(\underline{m}) \otimes x$ in $\left(\otimes_{R}^{b} M\right) \otimes_{R} N$ is nonzero and is annihilated by $I(M)$ and hence is in the torsion submodule; this is where the hypothesis that $I(M)$ contains a nonzerodivisor is used.

(2) We claim that there exists a syzygy relation (2.2.1) with $\underline{m}$ a minimal generating set for $M,(\underline{r}) \subseteq \mathfrak{m}$, and some $r_{i}$ a nonzerodivisor. 
Indeed, $\nu_{R}(M) \geq b$ since $M$ is not free. Choose elements $m_{1}, \ldots, m_{b}$ that form part of a minimal generating set for $M$ and such that $m_{1}, \ldots, m_{b-1}$ form a basis for $\mathrm{Q}(R) \otimes_{R} M$ over $\mathrm{Q}(R)$. Then there is a syzygy relation as in (2.2.1) in which $r_{b}$ is a nonzerodivisor.

The element $\tau(\underline{m})$ in $\otimes_{R}^{b} M$ is annihilated by $(\underline{r})$, by Proposition 2.2, and is not in $\mathfrak{m}\left(\otimes_{R}^{b} M\right)$, by Lemma 2.6. Since $(\underline{r})$ has a nonzerodivisor, it follows as in (1) that the torsion submodule of $\left(\otimes_{R}^{b} M\right) \otimes_{R} N$ is nonzero.

We learned recently that in 2011, in response to a query on MathOverflow, David Speyer gave a proof in [13] of (1) that is quite similar to ours when $R$ is a domain.

One cannot always expect torsion in tensor powers of nonfree modules, as the following shows.

EXAmPLE 2.8. Let $R=k[[x, y]] /(x y)$, where $k$ is a field. The torsion-free $R$-module $M:=R /(x)$ is not free; however, $\otimes_{R}^{n} M$ is isomorphic to $R /(x)$ for every $n \geq 1$ and hence is torsion-free.

The preceding results bring to the fore the following question:

Question 2.9. Let $R$ be a local domain. Is there an integer $b$, depending only on $R$, such that $\otimes_{R}^{n} M$ has torsion for every finitely generated nonfree $R$-module $M$ and every integer $n \geq b$ ?

The condition that $R$ be a domain is to avoid the situation of Example 2.8. When $R$ is regular, one can take $b=\operatorname{dim} R$, by results of Auslander [1, Theorem 3.2] and Lichtenbaum [10, Corollary 3].

\section{§3. Torsion "carriers"}

Some modules, even though they are torsion-free, usually generate torsion in tensor products. For example, over a local ring $(R, \mathfrak{m}, k)$ of positive depth, the maximal ideal $\mathfrak{m}$ is such a module: for any finitely generated nonfree $R$-module $M$, the tensor product $\mathfrak{m} \otimes_{R} M$ has torsion. To see this, observe that the short exact sequence

$$
0 \rightarrow \mathfrak{m} \rightarrow R \rightarrow k \rightarrow 0
$$

yields an injection from the torsion module $\operatorname{Tor}_{1}^{R}(k, M)$ into $\mathfrak{m} \otimes_{R} M$; moreover, $\operatorname{Tor}_{1}^{R}(k, M) \neq 0$ because $M$ is not free.

We give two more examples of torsion carriers: the integral closure $\bar{R}$ of a 1-dimensional analytically unramified ring $R$, and the Frobenius powers 
$\varphi^{e} R$ of a complete intersection $R$ of characteristic $p$. Recall that a local ring is analytically unramified provided that its completion is reduced. If $R$ is 1-dimensional, an equivalent condition is that $R$ be Cohen-Macaulay with finitely generated integral closure $\bar{R}$ (see [9, Theorem 4.6]).

THEOREM 3.1. Let $R$ be a 1-dimensional analytically unramified local ring, and let $\bar{R}$ be the integral closure of $R$ in its total quotient ring. If $M$ is a finitely generated $R$-module for which $\bar{R} \otimes_{R} M$ is torsion-free, then $M$ is free.

Proof. Let $\mathfrak{p}_{1}, \ldots, \mathfrak{p}_{s}$ be the minimal prime ideals of $R$, and for each $i$ let $r_{i}$ be the dimension of the $R_{\mathfrak{p}_{i}}$-vector space $M_{\mathfrak{p}_{i}}$. Put $n=\nu_{R} M$, the minimal number of generators of the $R$-module $M$, and choose an exact sequence

$$
0 \rightarrow K \rightarrow R^{(n)} \rightarrow M \rightarrow 0
$$

If we can show that $r_{i}=n$ for each $i$, we will know that $K$ is torsion and hence zero, and we will be done.

Put $D_{i}=\overline{R / \mathfrak{p}_{i}}$, the integral closure of the domain $R / \mathfrak{p}_{i}$. Since $R$ is reduced, we have inclusions

$$
R \hookrightarrow \prod_{i=1}^{s} R / \mathfrak{p}_{i} \hookrightarrow \prod_{i=1}^{s} D_{i} \hookrightarrow \prod_{i=1}^{s} \mathrm{Q}\left(R / \mathfrak{p}_{i}\right)=\mathrm{Q}(R) .
$$

We see that $\bar{R}=\prod_{i=1}^{s} D_{i}$; moreover, each $D_{i}$ is a semilocal Dedekind domain and therefore a principal ideal domain. Since $\bar{R} \otimes_{R} M$ is torsion-free, it is projective, in fact free of rank $r_{i}$ on the component $D_{i}$. Therefore, setting $e_{i}=\nu_{R} D_{i}$, we have the equations

$$
r_{1} e_{1}+\cdots+r_{s} e_{s}=\nu_{R}\left(\bar{R} \otimes_{R} M\right)=\left(\nu_{R} \bar{R}\right) \cdot\left(\nu_{R} M\right)=\left(e_{1}+\cdots+e_{s}\right) n .
$$

Since $r_{i} \leq n$ for each $i$, it follows from these equations that $r_{i}=n$ for each $i$.

Let $R$ be a Noetherian ring of positive characteristic $p$, and let $\varphi: R \rightarrow R$ be the Frobenius endomorphism $r \mapsto r^{p}$. Given an $R$-module $M$ and a positive integer $e$, we write ${ }^{e} M$ for the $R$-module obtained from $M$ by restriction of scalars along $\varphi^{e}$; thus, $r \cdot m=r^{p^{e}} m$ for $r \in R$ and $m \in M$. Observe that $M$ is torsion-free if and only if $\varphi^{e} M$ is torsion-free for some (equivalently, all) $e \geq 1$. Following [12], we write $F^{e}(M)$ for the tensor product $M \otimes_{R} \varphi^{e} R$. One views $F^{e}(M)$ as a right $R$-module: the action of $R$ on $F^{e}(M)$ 
comes from the right (ordinary) action of $R$ on $\varphi^{e} R$. Thus, $F^{e}(R) \cong R$ as $R$-modules, and it follows that $F^{e}(M)$ is finitely generated if $M$ is finitely generated.

THEOREM 3.2 ([12, Corollaire 1.10]). Let $R$ be a local ring of characteristic $p$, and let $M$ be a finitely generated $R$-module. If $M$ has finite projective dimension, then $\operatorname{Tor}_{i}^{R}\left(M, \varphi^{e} R\right)=0$ for all $e \geq 1$ and all $i \geq 1$.

The converse of Theorem 3.2 is true and was proved by Herzog [5, Theorem 3.1]. For complete intersections, the following strong converse was proved by Avramov and Miller.

Theorem 3.3 ([3, Main Theorem $])$. Let $(R, \mathfrak{m})$ be a complete intersection of characteristic $p$, and let $M$ be a finitely generated $R$-module. If $\operatorname{Tor}_{i}^{R}\left(M, \varphi^{e} R\right)=0$ for some $e \geq 1$ and some $i \geq 1$, then $M$ has finite projective dimension.

The proof that $(1) \Longrightarrow(2)$ in the next theorem follows many of the same steps Auslander used in [1, proof of Lemma 3.1]. The main differences are that we have to allow for the possibility that $\varphi^{e} R$ is not finitely generated and that we appeal to Theorems 3.2 and 3.3 for a replacement of rigidity of Tor over regular local rings. Recall that a module $M$ is generically free provided that $M_{\mathfrak{p}}$ is a free $R_{\mathfrak{p}}$-module for each $\mathfrak{p} \in$ Ass $R$.

THEOREM 3.4. Let $(R, \mathfrak{m})$ be a complete intersection of characteristic $p$, and let $M$ be a finitely generated, generically free $R$-module. Fix a positive integer e. The following conditions are equivalent:

(1) $F^{e}(M)$ is torsion-free, and

(2) $M$ is torsion-free and of finite projective dimension.

Proof. Suppose that (1) holds, and apply $-\otimes_{R} \varphi^{e} R$ to the short exact sequence (2.4.3), getting an exact sequence

$$
F^{e}\left(\top_{R} M\right) \stackrel{\alpha}{\longrightarrow} F^{e}(M) \stackrel{\beta}{\longrightarrow} F^{e}\left(\perp_{R} M\right) \rightarrow 0 .
$$

Since $F^{e}\left(\top_{R} M\right)$ is torsion and $F^{e}(M)$ is torsion-free, we see that $\alpha=0$, whence $\beta$ is an isomorphism. In particular, $F^{e}\left(\perp_{R} M\right)$ is torsion-free. Next, consider the universal pushforward (see [6, Section 1]):

$$
0 \rightarrow \perp_{R} M \rightarrow R^{(m)} \rightarrow N \rightarrow 0 .
$$


Applying $-\otimes_{R}{ }^{e} R$ to this sequence, we obtain an injection

$$
\operatorname{Tor}_{1}^{R}\left(N, \varphi^{e} R\right) \hookrightarrow F^{e}\left(\perp_{R} M\right)
$$

Now $\perp_{R} M$ is clearly generically free, and from the construction of the universal pushforward in [6, Section 1], one checks that $N$ is generically free as well. It follows that $\operatorname{Tor}_{1}^{R}\left(N, \varphi^{e} R\right)$ is torsion. Since $F^{e}\left(\perp_{M}\right)$ is torsion-free, we have $\operatorname{Tor}_{1}^{R}\left(N, \varphi^{e} R\right)=0$. Now we invoke Theorems 3.2 and 3.3 to see that

$$
\operatorname{Tor}_{i}^{R}\left(N, \varphi^{e} R\right)=0 \text { for all } i \geq 1
$$

and, moreover, that $N$ has finite projective dimension. From (3.4.1) it follows that $\operatorname{Tor}_{i}^{R}\left(\perp_{R} M, \varphi^{e} R\right)=0$ for all $i \geq 1$ and that $\perp_{R} M$ has finite projective dimension. Therefore, we will have (2) once we show that $\top_{R} M=0$. For this, we apply $-\otimes_{R} \varphi^{e} R$ once again to (2.4.3), to get an injection

$$
F^{e}\left(\top_{R} M\right) \hookrightarrow F^{e}(M) .
$$

Since $F^{e}\left(\top_{R} M\right)$ is torsion and $F^{e}(M)$ is torsion-free, we have $F^{e}\left(\top_{R} M\right)=0$. If $\top_{R} M$ were nonzero, there would be a surjection $\top_{R} M \rightarrow R / \mathfrak{m}$. But then $F^{e}(R / \mathfrak{m})=0$, that is, $\mathfrak{m}^{\varphi^{e}} R=\varphi^{e} R$, an obvious contradiction, since $\mathfrak{m}^{\varphi^{e}} R \subseteq \mathfrak{m}$. Thus $\top_{R} M=0$, and the proof that $(1) \Longrightarrow(2)$ is complete.

Now assume that (2) holds. Since $M$ is torsion-free, we can build the universal pushforward (see [6, Section 1]):

$$
0 \rightarrow M \rightarrow R^{(\nu)} \rightarrow N \rightarrow 0
$$

where $\nu=\nu_{R} M^{*}$. Then $N$ has finite projective dimension. Now Theorem 3.2 implies that $\operatorname{Tor}_{i}^{R}\left(N, \varphi^{e} R\right)=0$ for all $i \geq 1$. Therefore, $\operatorname{Tor}_{1}^{R}\left(M, \varphi^{e} R\right)=0$, and we get an injection $F^{e}(M) \hookrightarrow\left(\varphi^{e} R\right)^{(\nu)}$, whence $F^{e}(M)$ is torsion-free.

From Theorem 3.2 (alternatively, from the proof of Theorem 3.4), we get Tor independence (item (2) in the Introduction), as follows.

Corollary 3.5. If $R$ and $M$ satisfy the equivalent conditions of Theorem 3.4, then $\operatorname{Tor}_{i}^{R}\left(M, \varphi^{e^{\prime}} R\right)=0$ for every $i \geq 1$ and every $e^{\prime} \geq 1$.

Of course, if $M$ is torsion-free, the converse of Corollary 3.5 holds, by Theorem 3.3. In fact, it suffices to check that $\operatorname{Tor}_{i}^{R}\left(M, \varphi^{e^{\prime}} R\right)=0$ for a single $e^{\prime}$ and a single $i$. 
Recall that $R$ is F-finite provided that $\varphi$ is a finite map, that is, that $R$ is module-finite over $\varphi(R)$. In this case, $\varphi^{e}$ is a finite map for each $e \geq 1$. Note that the action of $R$ on the module $\left(\varphi^{e} M\right)$ in items (1) and (2) below is the Frobenius action $m \cdot r=m r^{p^{e}}$.

Corollary 3.6. Assume that $(R, \mathfrak{m})$ is a reduced local ring, is F-finite, and is a complete intersection. The following conditions are equivalent:

(1) $F^{e}\left(\varphi^{e^{\prime}} M\right)$ is torsion-free for every torsion-free $R$-module $M$ and every pair e, $e^{\prime}$ of positive integers;

(2) $F^{e}\left(\varphi^{e^{\prime}} M\right)$ is torsion-free for some nonzero finitely generated $R$-module $M$ and some pair e, $e^{\prime}$ of positive integers; and

(3) $R$ is regular.

Proof. Obviously (1) $\Longrightarrow(2)$, and the implication $(3) \Longrightarrow(1)$ holds by Kunz's theorem [8, Theorem 2.1] that the $R$-module ${ }^{\varphi^{e}} R$ is flat when $R$ is regular.

To prove that $(2) \Longrightarrow(3)$, we note that $\varphi^{e^{\prime}} M$ is a finitely generated $R$ module, by F-finiteness. Also, $\varphi^{e^{\prime}} M$ is generically free because $R$ is reduced. By Theorem 3.4, ${ }^{\varphi^{\prime}} M$ has finite projective dimension, and now [2, Theorem 1.1] implies that $R$ is regular.

Acknowledgments. We thank the referees for their critical reading of the manuscript.

Part of this material is based upon work supported by National Science Foundation grant DMS-0932078 while the second and fourth authors were in residence at the Mathematical Sciences Research Institute in Berkeley, California, during the autumn of 2013. The second author was partly supported by NSF grant DMS-1201889 and a Simons Fellowship, and the fourth author was partly supported by a Simons Collaboration Grant.

\section{REFERENCES}

[1] M. Auslander, Modules over unramified regular local rings, Illinois J. Math. 5 (1961), 631-647. MR 0179211.

[2] L. L. Avramov, M. Hochster, S. B. Iyengar, and Y. Yao, Homological invariants of modules over contracting endomorphisms, Math. Ann. 353 (2012), 275-291. MR 2915536. DOI 10.1007/s00208-011-0682-z.

[3] L. L. Avramov and C. Miller, Frobenius powers of complete intersections, Math. Res. Lett. 8 (2001), 225-232. MR 1825272. DOI 10.4310/MRL.2001.v8.n2.a10.

[4] W. Bruns and J. Herzog, Cohen-Macaulay Rings, Cambridge Stud. Adv. Math. 39, Cambridge University Press, Cambridge, 1993. MR 1251956. 
[5] J. Herzog, Ringe der Charakteristik p und Frobeniusfunktoren, Math. Z. 140 (1974), 67-78. MR 0352081.

[6] C. Huneke, D. A. Jorgensen, and R. Wiegand, Vanishing theorems for complete intersections, J. Algebra 238 (2001), 684-702. MR 1823780. DOI 10.1006/jabr.2000.8603.

[7] C. Huneke and R. Wiegand, Tensor products of modules, rigidity and local cohomology, Math. Scand. 81 (1997), 161-183. MR 1612887.

[8] E. Kunz, Characterization of regular local rings for characteristic p, Amer. J. Math. 91 (1969), 772-784. MR 0252389.

[9] G. J. Leuschke and R. Wiegand, Cohen-Macaulay Representations, Math. Surveys Monogr. 181, Amer. Math. Soc., Providence, 2012. MR 2919145. DOI $10.1090 /$ surv/181.

[10] S. Lichtenbaum, On the vanishing of Tor in regular local rings, Illinois J. Math. 10 (1966), 220-226. MR 0188249.

[11] S. Mac Lane, Homology, reprint of the 1975 original, Springer, Berlin, 1995. MR 1344215.

[12] C. Peskine and L. Szpiro, Dimension projective finie et cohomologie locale, applications à la démonstration de conjectures de M. Auslander, H. Bass et A. Grothendieck, Publ. Math. Inst. Hautes Études Sci. 42 (1973), 47-119. MR 0374130.

[13] C. Speyer, Torsion-free tensor powers, preprint, http://mathoverflow.net/ questions/73120/torsion-freetensor-powers (accessed 11 June 2015).

Olgur Celikbas

Department of Mathematics

University of Missouri

Columbia, Missouri 65211

USA

Srikanth B. Iyengar

Department of Mathematics

University of Nebraska

Lincoln, Nebraska 68588-0130

USA

Greg Piepmeyer

Columbia Basin College

Pasco, Washington 99301

USA

Roger Wiegand

Department of Mathematics

University of Nebraska

Lincoln, Nebraska 68588-0130

USA 